\title{
33. GROUPE DU TRAVAIL SUR REGIONS CHOISIES, 23 ET 24 SESSION MIXTE
}

\author{
Report of Meeting, 29 August 1967
}

PRESIDENT: B. J. Bok.

SECRETARY: K. Loden.

At the request of Vasilevskis and Murray, the Presidents of Commissions 23 and 24 planned a Joint Meeting of the two Commissions, together with the Working Group on Selected Areas, for the purpose of discussing the problems that arise in the planning for future large-scale astrometric programs of proper motions.

In his Introductory Remarks, Murray reminded the Meeting that modern techniques of automatic measurement of photographic plates for stellar positions, coupled with new and efficient methods of data processing (reductions and analysis) are making it possible to measure very large numbers of stars on many plates with early epochs. Such measurements can be combined, for the determinations of mass proper motions, with results from recent plates. The Kapteyn Selected Areas hold special promise for work on faint stars, since for these centers large numbers of excellent earlyepoch plates are available. It is essential that astronomers and observatories interested in the work should coordinate their efforts. Murray spoke specifically about the present plan of Royal Greenwich Observatory to remeasure the proper motions for the stars in the Radcliffe Catalogue of Selected Areas. The 26-inch Refractor at Herstmonceux seems ideally suited for obtaining the second-epoch plates.

Vasilevskis gave a survey of the Lick proper motion program, in which the proper motions are referred to galaxies. This program consists of 1246 fields covering the sky from $\delta=+90^{\circ}$ to $\delta=-23^{\circ}$. The instrument is the $20^{\prime \prime}$ Carnegie Astrograph. The fields are of size $6^{\circ} \times 6^{\circ}$, on $17^{\prime \prime} \times$ $17^{\prime \prime}$ plates, which gives a scale of $55^{\prime \prime} .14$ per $\mathrm{mm}$. The first epoch plates have been taken with a blue (photographic) lens in 1947-1954. The second epoch plates will be taken in 1967 and onwards in two colors, blue and yellow. The final catalogue will contain proper motions, magnitudes and colors. The limiting magnitude will be approximately $18^{m} .5$. The brightest limit is $7^{m} .5$. All plates will be measured automatically. There will be two initial phases of the project: First, about 150 fields will be treated in order to develop the best routines; Second, the measurements for all fields will be repeated after $1-2$ years.

During the discussion that followed the presentations, the southern hemisphere needs were stressed. The new Yale-Columbia Program in Argentina will ultimately extend the Lick Program to far southern declinations, but it will be many years before second-epoch plates with sufficiently long base lines in time can be obtained. The possibility should be explored of obtaining second-epoch plates for the parallax centers of the original Johannesburg, Yale-Columbia, Program, initiated by F. Schlesinger. The 26-inch Yale-Columbia Refractor is now the property of Mount Stromlo Observatory and the second-epoch plates might well be obtainable with the original instrument. It was recommended that any future Working Group should examine how and where these secondepoch plates might be photographed and measured.

It was further noted that many good early collections of northern and southern Selected Area photographs are available at the Harvard, Yale, Mount Wilson, Pulkovo and other Observatoriesand that there is also a fine collection of early Selected Area plates at the Kapteyn Astronomical Laboratory. These can be important sources for first-epoch plates for the measurement of proper motions of faint stars.

Plaut summarized the Yale-Columbia program of southern Selected Areas. There are first-epoch plates available for all southern areas and second-epoch plates for the declination zone $-15^{\circ}$. This latter zone has been measured differentially at Groningen but the reductions are not yet finished. 
The second-epoch plates were taken in the early 1930's. If a new series of plates were taken in the near future, then the weight of the results would be increased four-fold. It is obviously important that this be done.

Reference was also made to the northern Selected Area needs and prospects. Vasilevskis mentioned that Lick Observatory has a special series of first-epoch plates, made with the Carnegie Astrograph, for all Selected Areas north of $-15^{\circ}$. There are no immediate plans for second-epoch plates, nor for the measurement of the Selected Area material, but, if desired, preference might be given to some of the Selected Area centers. Murray stressed the importance of having available at the earliest possible date absolute (Lick) proper motions for 30 to 40 stars, which would then serve as standards for the reduction of measurements from other Selected Area photographs. It would then become possible to reduce all Selected Area relative proper motions to absolute values on the Lick system referred to faint galaxies. Luyten stressed the importance of star-like quasars as reference objects for the measurement of stellar proper motions.

Luyten spoke briefly about the Minnesota Program for the detection of stars with proper motions in excess of 0 ". 1 per year and about the measurements of the proper motions for the faint stars of this sort. 48-inch Palomar-Schmidt plates, covering 40 square degrees each, are searched, and large proper motion stars are found at the average rate of 12 per square degree. An automatic measuring engine is under construction and all reductions will be by modern data-processing techniques.

The difference between the epochs will be 14-17 years. The magnitude interval is $4^{m}-21^{m}$ and the mean error is \pm 0 ". 020 . The accuracy for the faint stars is better than for the bright ones. A reduction to absolute proper motions by means of quasars will be performed; it is also intended to have normal galaxies as a reference system. Luyten mentioned that Schmidt telescopes are good for astrometric work, but that the measurements should preferably be limited to stars relatively near the center of the plate.

Blaauw spoke briefly about the new McCormick Observatory proper motion survey. Present plans call for a repetition of the Vyssotsky-Williams program under a joint program involving the McCormick Observatory and the Kapteyn Astronomical Laboratory (Blaauw). The original plates are all centered on bright (parallax) stars, dimmed to $V=10.5$ or fainter with the aid of a rotating sector. The first-epoch plates go back to the period 1915 to 1930, and these are to be repeated once again, thus providing for a very long base-line in time. Since the central stars ( 700 fields) all have known proper motions on the FK4 system, absolute proper motions of high precision and on the FK4 system can be found for the 30000 faint stars. In addition to the proper motion measurements an effort will be made to obtain for these stars precise photometric data and, possibly, also objectiveprism radial velocities. Blaauw urges improved meridian circle observations for the central stars in the $700 \mathrm{McC}$ Cormick fields.

In conclusion, the Meeting (with 43 in attendance) went on record as favoring the creation of a Working Group on Proper Motions of Faint Stars, with Plaut as Chairman, and as members: A.N. Deutsch, Dieckvoss, T. Elvius, Luyten, McCuskey, Murray and Vasilevskis. 